\title{
Extension, Stress and Composite Failure in Bedded Rock Masses
}

\author{
J.V. Simmons Sherwood Geotechnical and Research Services, Australia \\ P.J. Simpson BHPBilliton Mitsubishi Alliance Coal Pty Ltd, Australia
}

\begin{abstract}
The mechanism of composite deformation in open pit coal mine slopes combines slip along existing defects with strength mobilisation involving rock material. Mining activity and initial stress conditions are linked with the onset of relatively sudden, unpredicted failures. In order to develop more reliable methods for recognising potential failure and providing early warning signs, it is first necessary to identify and understand the potential trigger processes. This paper describes early progress in numerical modelling of bedded coal measures rock masses under conditions where composite slope failures have occurred repeatedly. Results to date illustrate the effects of initial stress on extensional deformations within a highwall slope. The results are linked to observations of actual failures, and highlight the role of extension strain fracturing near the slope toe as a triggering mechanism.
\end{abstract}

\section{Introduction}

Composite slope failures in sedimentary rock masses often show evidence of defects that dip steeply outwards from the slope face. Such structures are not always recognised in advance, and are also not continuous and persistent enough to generate kinematically-controlled failure. Irrespective of the orientations of the typical lithological joints within the bedded rock mass, the steep-dipping defects are always oriented towards the excavation. This raises the prospect of defects being generated by the excavation-induced process leading to failure. To investigate this phenomenon finite element modelling was undertaken using a commercially available package in such a manner that some effects of blockiness and rock mass behaviour could be simulated in addition to the contrasting properties of the bedded structure. Measurements of in situ stress were used to set bounds on the possible stress states affecting response to excavation. Stacey's (1981) Extension Strain Fracture Criterion (ESFC) was used to investigate the pre-failure processes near the slope toe. The model results indicate that extension fracture growth is almost inevitably predicted and would potentially develop into larger-scale structures similar to those observed. Further investigations are underway to determine whether fracture growth phenomena could be used to help predict failure conditions.

\section{Previous experience and composite failure mechanism hypothesis}

This paper describes the continuation of investigations reported by Simmons and Simpson (2006). Largescale and apparently sudden open pit highwall failures have occurred in many of northeastern Australia's Bowen Basin coal mines. While it is possible that rigorous surveillance of normal highwall conditions may have been able to detect small precursor movements for some of the reported failures, such a level of monitoring is not typically undertaken for practical reasons. The incidence of such failures is reportedly very low but the potential consequences may be extremely high because of the lack of obvious warning signs combined with in-pit mining activity.

Common features of these failures include tectonic overprint structures which were often not recognised prior to failure, lack of relationship between wall height or scale of failure and lithological conditions, and variable stand-up time but commonly a coincidence of failure with mining activity at or near pit floor level. Simmons and Simpson (2006) reported that traditional limit equilibrium stability analysis tools, rock tests, defect measurements, and observations of rock mass conditions were not generally helpful for identifying and predicting composite failures. On the other hand, it appeared that extensional straining, brittle fracture processes, and relatively high tectonic stresses played a significant role in development of such failures. Slope monitoring radar measurements indicate that movement rates increase when mining within about $15 \mathrm{~m}$ of highwall toes, but are nevertheless too small to be recognised by visual observation. 
Several lines of investigation by others support the authors' opinion that stress conditions and deformation patterns within rock slopes must be better understood before it will be possible to recognise and predict composite failure conditions. Dight (2006) described observations monitoring and numerical modelling in support of the view that extensional deformations and stress conditions lead to the development of new structures that control the location and magnitude of failure conditions in rock slopes. Dight discussed the potential for coalescence of small extensional fractures within rock material to create large-scale defects along which shear deformation is concentrated. Such a process would go a long way towards explaining some of the characteristics of composite failures reported by Simmons and Simpson (2006). Stacey (2006) described experiments with physical modelling of jointed and bedded rock masses to show how extensional deformation may mobilise variable tensile resistance within the mass depending on geometric features of the slope and blockiness of the rock mass fabric.

Concepts of extensional straining and tensile fracture development within the rock mass would also help explain why composite failure mechanisms can develop with so little warning even in medium strength wellbedded and well-jointed rock masses. In a stress-controlled situation such as an unsupported rock slope, the onset of runaway brittle fracture would result in rapid collapse.

Rock material failure has been the subject of considerable research as it is amenable to well-controlled laboratory experimentation. Rock materials of at least medium strength typically fail by longitudinal splitting under uniaxial stress conditions. There is a transition from brittle to ductile deformation response with increasing confining pressure accompanied by localisation of tensile microfractures into specimen-scale shear surfaces and eventually into non-localised shear straining. Mogi (2007) provided evidence that the transition from brittle fracture to ductile yielding for silicate rocks occurs at a principal stress condition where $\sigma_{1} / \sigma_{3} \geq 4$.4. From the results of experiments Mogi concluded that brittle behaviour forms a discrete shear fracture surface in a plane perpendicular to the intermediate principal stress $\left(\sigma_{2}\right)$ direction and thus depends only on the values of $\sigma_{1}$ and $\sigma_{3}$, whereas ductile yielding is less direction-specific and requires consideration of all three principal stress values.

Stacey (1981) proposed a simple extension strain fracture criterion (ESFC) for the purpose of predicting the extent of the zone of failure around an excavation in brittle rock. This was based on observations of the onset of inferred microfracture growth in laboratory tests as well as on elasticity-based back analysis of excavated stress states. The ESFC concept has been widely misinterpreted as a rock failure criterion, and possibly for this reason has not been as widely accepted for what it should be. Fujii et al (1998) presented evidence that a similar extension strain-based criterion may be used for rock failure prediction, but other researchers (e.g. quoted in Dight 2006) prefer a prediction of brittle failure conditions based on principal stress conditions.

Coal measures rock masses consist of bedded sediments with various classes of jointing dependent on stress history. For burial and uplift without the influence of pore fluid pressures, two sets of orthogonal joints of limited continuity and persistence typically develop approximately perpendicular to bedding partings. Such lithological joints can be separately identified from more persistent joints where pore fluid pressures are elevated to the extent that hydraulic fracture becomes a consideration (Mandl, 2005). Where tectonic history is more complex, involving folding or thrusting, defect patterns within the rock mass become correspondingly complex and include obvious faults, less obvious wrenching, and generation of tensile fractures in principal extension directions that may have been generated within larger-scale shear deformation zones.

For eastern Australia which includes the major coalfields, virgin stress fields are widely observed to have the minor principal stress $\sigma_{3}$ in the approximately vertical direction with the maximum and intermediate stresses $\sigma_{1}$ and $\sigma_{2}$ within the approximately horizontal plane. The directions of the horizontal principal stresses vary with location according to continental crust-scale processes (Hillis et al, 1999). It is not unusual for in situ stress measurements within the Bowen Basin to indicate lateral stress ratios (i.e. of $\sigma_{1} / \sigma_{3}$ ) of 2 or greater. Within several Bowen Basin open pit coal mines, observations of lipping and offsets of vertical features suggest that there are zones bounded by wrenches where horizontal stresses are particularly high. It is generally in such high stress areas that composite failure mechanisms occur with greater frequency.

After considering the above, the authors have formed the hypothesis that composite failures in bedded rock masses are most likely to be experienced where initial ground stresses are relatively high, and most likely to develop in conditions where extensional straining causes fracture growth leading to formation or 
enhancement of shear fractures that are dipping steeply outwards towards the pit. Natural blockiness of tectonically overprinted rock masses makes the concentration of stresses more likely. Removal of the final stage of the highwall toe support, by coal mining or floor preparation activity, is therefore likely to provide the final trigger for fracture growth leading to wall collapse.

\section{Case history and numerical modelling objectives}

Saraji Mine is operated by BMA Coal and is located in the northern sector of the Bowen Basin. It has been producing high quality metallurgical coal since 1974 using dragline strip mining assisted by shovel/truck prestripping. The Ramp 12 area of the mine has a history of inferred high stress conditions evidenced by lipping and offsets and has experienced several composite highwall failures. It is also the area where the significance of steep outward-dipping defects for potential highwall failure was first recognised by BMA Coal. Stress measurements have been made in this area using hydraulic fracture and overcoring techniques. The area was identified in 2006 as a site for detailed investigations of highwall response to overburden stripping and coal mining. Mining of the second pass of coal from Strip 23 was completed in mid-January 2007 and images of the highwall were acquired for interpretation of rock mass defects. Heavy rainfall at the end of January triggered a composite highwall failure in the area that had been previously identified for the field experiment site. Figure 1 shows the failure area immediately after the rain event. Both margins of the failed area were defined by highly continuous and persistent joints.

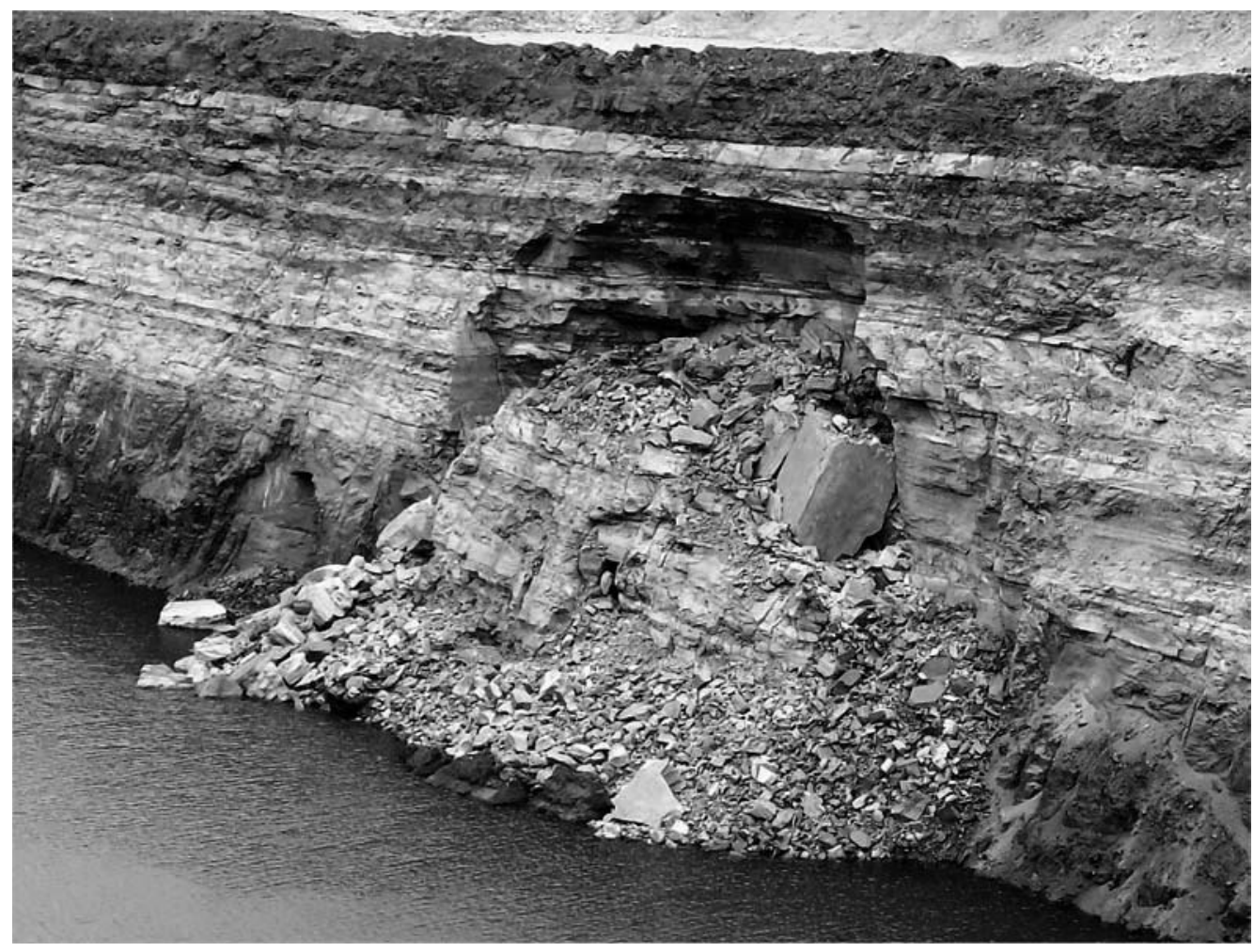

Figure 1 Composite failure of Saraji Mine Ramp 12 Strip 23 highwall January 2007 triggered by heavy rain shortly after coal removal

The goal of the field investigations was to complement numerical modelling of excavations in bedded rock typical of coal measures conditions where composite failures had been experienced. The occurrence of the Strip 23 failure provided an ideal background for modelling of conditions that were known to have contributed to a failure.

The target Dysart seam in the Strip 23 is located at a depth of approximately $100 \mathrm{~m}$ below original natural surface. Because of sequential prestripping and mining of the overlying $\mathrm{K}$ seam, the Dysart pass highwall height is approximately $45 \mathrm{~m}$ from crestline to seam floor. Neither the overall depth to floor nor the highwall height are unusual for Bowen Basin conditions, and mines with much higher faces and deeper floors have 
not experienced Saraji Mine's Ramp 12 incidence of composite failures. The objectives of numerical modelling of the highwall were therefore to build a routine numerical model using commercially available software, to replicate the sequence of stripping and coal mining, and to examine the influence of initial stress state on the response of the highwall.

\section{Rock material and rock mass conditions}

Routine coal mine exploration drilling is carried out for resources definition using open drillholes with cutting logged by geologists but also using a suite of geophysical tools for continuous material definition. Partially or fully cored holes for geotechnical data gathering purposes are comparatively rare, except when development project studies are undertaken. For the Saraji Ramp 12 area there have been several geotechnical coreholes drilled, with a reasonable cross-section of UCS and density tests for most geological units within the overburden sequence. Reasonable correlations have been developed by BMA Coal between sonic velocity or density and UCS. Occasional face mapping has been undertaken so that there is at least a useful indication of the rock mass defect fabric for the typical overburden conditions shown in Figure 1.

Table 1 is a summary of the rock mass units identified for the $\mathrm{K}$ - Dysart interburden and floor sequence in the Ramp 12 area. The sequence can be summarised as interbedded siltstone-sandstone (Unit 1) overlying massive sheeted sandstone and/or current bedded sandstone (Unit 2), in turn overlying carbonaceous siltstone (Unit 3) that forms the roof for the Dysart upper seam plies. The Dysart upper seam (DYU) is separated from the lower seam (DYL) by a silty fine grained sandstone parting and within the lower seam are several carbonaceous siltstone bands and a thin puggy clay band. The floor of the Dysart lower plies is a thinly bedded low-medium strength silty sandstone (Unit 4). Lipping and offsets are frequently observed at the contact between Units 2 and 3, and at the Unit 3 - DYU roof contact. At these two surfaces, as well as at the DYU - parting contact and at the DYL puggy clay band, there is evidence of shearing.

Table 1 Rock mass units forming $\mathrm{K}$ - Dysart seam interburden and floor sequence

\begin{tabular}{|c|c|c|c|}
\hline Unit & Description & Thickness Range (m) & Model Thickness (m) \\
\hline 1 & $\begin{array}{l}\text { Interbedded siltstone-sandstone (medium - } \\
\text { strength), medium grey }\end{array}$ & $10-20$ & 15 (at highwall) \\
\hline 2 & $\begin{array}{l}\text { Massive sheeted sandstone and/or current- } \\
\text { bedded sandstone (medium - high and in } \\
\text { places very high strength), light grey }\end{array}$ & $15-25$ & 20 \\
\hline 3 (roof) & $\begin{array}{l}\text { Massive to thick bedded carbonaceous } \\
\text { siltstone (medium - high strength), dark grey } \\
\text { to black }\end{array}$ & $5-20$ & 10 \\
\hline $\begin{array}{l}\text { DYU } \\
\text { coal }\end{array}$ & $\begin{array}{l}\text { Banded bright high rank bituminous coal, } \\
\text { finely cleated, medium strength }\end{array}$ & 3 & 5 \\
\hline parting & $\begin{array}{l}\text { Thin-bedded fine grained sandstone, medium } \\
\text { strength, medium grey }\end{array}$ & $0.8-2.4$ & $\begin{array}{l}\text { incorporated into } \\
\text { DYL }\end{array}$ \\
\hline $\begin{array}{l}\text { DYL } \\
\text { coal }\end{array}$ & $\begin{array}{l}\text { Laminated bright and dull high rank } \\
\text { bituminous coal, low strength, containing } \\
\text { puggy high plasticity clay band }\end{array}$ & 2 & $2 \times 2.5$ \\
\hline 4 (floor) & $\begin{array}{l}\text { Thin-bedded silty sandstone, low strength, } \\
\text { medium strength, medium grey }\end{array}$ & $20-30$ & 50 \\
\hline
\end{tabular}

The seam structure dip in the Ramp 12 area is typically about $4^{\circ}$ towards the east so that the strips run approximately north-south. Fully persistent face-high near-vertical joints intersect the highwall obliquely at two orientations, while a third less persistent near-vertical joint is approximately parallel to the highwall orientation. The spacing of one of the oblique sets varies from a typical value of $5 \mathrm{~m}$ to zones where it is less than $100 \mathrm{~mm}$. The latter appear to form boundaries between blocks with different lateral stress intensities, judging from the amount of lipping to either side of such zones. The coal measures in the Saraji area are 
known to have been deformed by thrusting, and the near-vertical fracture zones are believed to be wrench zones between parcels of thrusted material gliding on shear surfaces at or near the Dysart seam horizon.

Limited core log information and the lack of systematic face mapping mean that there were no reliable measurements of rock mass characteristics that could be used to estimate rock mass strength and stiffness from material tests. While BMA Coal had well-established relationships between geophysical measurements and both strength and density of rock material, no such relationships have been developed for rock mass parameters. Table 2 is a summary of the rock material parameters based on results of approximately 170 tests on core samples.

Table 2 Summary rock material parameters from laboratory tests on core samples

\begin{tabular}{|c|c|c|c|c|c|}
\hline Parameter & Unit 1 & Unit $2 \mathrm{a}^{(1,4)}$ & Unit $2 b^{(2)}$ & Unit 3 & Coal \\
\hline \multicolumn{6}{|c|}{ Moisture content (\%) } \\
\hline $\min$. & 1.90 & 1.30 & 1.10 & 1.90 & 0.90 \\
\hline $\max$. & 5.60 & 6.50 & 3.00 & 4.20 & 1.90 \\
\hline Average & 3.84 & 3.22 & 2.12 & 2.78 & 1.46 \\
\hline \multicolumn{6}{|c|}{ Density $\left(\mathrm{t} / \mathrm{m}^{3}\right)$} \\
\hline $\min$. & 2.324 & 2.128 & 2.419 & 2.282 & 1.296 \\
\hline $\max$. & 2.619 & 2.668 & 2.679 & 2.633 & 1.687 \\
\hline Average & 2.464 & 2.468 & 2.598 & 2.513 & 1.399 \\
\hline \multicolumn{6}{|c|}{$\underline{\mathrm{UCS}}(\mathrm{MPa})$} \\
\hline $\min$. & 10.6 & 7.2 & 62.3 & 17.9 & 9.2 \\
\hline $\max$. & 68.1 & 67.2 & 102.6 & 59.3 & 33.3 \\
\hline Average & 33.1 & 38.2 & 80.7 & 35.6 & 19.4 \\
\hline \multicolumn{6}{|c|}{ Young's modulus (GPa) } \\
\hline $\min$. & 1.72 & 3.69 & 10.50 & 1.33 & 3.60 \\
\hline $\max$ & 5.66 & 8.47 & 29.50 & 12.50 & 4.50 \\
\hline Average & 3.26 & 5.79 & 19.40 & 6.79 & 3.97 \\
\hline \multicolumn{6}{|c|}{ Poisson's ratio } \\
\hline $\min$. & 0.21 & 0.22 & 0.19 & 0.20 & $\mathrm{nd}^{(3)}$ \\
\hline $\max$. & 0.44 & 0.48 & 0.40 & 0.48 & nd \\
\hline Average & 0.35 & 0.29 & 0.28 & 0.26 & nd \\
\hline
\end{tabular}

Notes: (1) Unit 2a is massive sheeted clay-cemented lithic-quartz sandstone

(2) Unit $2 b$ is massive current-bedded calcite-cemented quartz-lithic sandstone co-deposited with Unit 2a

(3) no data on Poisson's ratio for coal samples

(4) no specific data on Unit 4 (floor) material, properties assumed to be equivalent to Unit 2a

To develop a computational model for the overburden conditions it was necessary to rely upon what is conveniently termed experience and engineering judgement. In addition, the ROCLAB ${ }^{\mathrm{TM}}$ code developed by RocScience was used to estimate rock mass parameters based upon the Generalised Hoek-Brown strength model and associated correlations with the empirical parameter GSI. The rock mass parameters that were adopted as the base-case condition for numerical modelling purposes are listed in Table 3.

Apart from plasticity properties and large-scale residual shear strength obtained from back analyses of instability mechanisms, very little is known about the geotechnical properties of the bedding-parallel weak surfaces within the interburden and seam sequence. For the purposes of modelling it was decided to include specific planes of weakness only where surfaces had formed part of observed instability mechanisms. These surfaces are located at or near the DYU - DYL interface and within the DYL ply sequence. The normal and shear stiffness parameters were based on experience, judgement, and advice regarding relationships between continuum and interface stiffnesses that were least likely to create numerical modelling convergence issues. The interface parameters that were adopted as the base-case condition for numerical modelling purposes are listed in Table 4. 
Table 3 Rock mass parameters for base-case numerical modelling

\begin{tabular}{|c|c|c|c|c|c|c|c|}
\hline Unit & $\gamma\left(\mathrm{kN} / \mathrm{m}^{3}\right)$ & $\mathrm{E}(\mathrm{MPa})$ & $v$ & $\mathrm{t}^{(1)}(\mathrm{MPa})$ & $\mathrm{c}_{\mathrm{p}, \mathrm{r}}^{(2)}(\mathrm{MPa})$ & $\phi_{\mathrm{p}, \mathrm{r}}^{(2)}$ & $\mathrm{D}^{(3)}$ \\
\hline 1 (i/b sst-slst) & 24.0 & 3260 & 0.20 & 0 & 0.169 & $37.6^{\circ}$ & 0 \\
\hline 2 (M sst) & 24.0 & 5560 & 0.20 & 0 & 0.445 & $55.3^{\circ}$ & 0 \\
\hline 3 (carb. slst) & 24.0 & 3500 & 0.20 & 0 & 0.556 & $44.0^{\circ}$ & 0 \\
\hline 4 (coal) & 14.0 & 2800 & 0.20 & 0 & 0.126 & $26.7^{\circ}$ & 0 \\
\hline 1 (blasted) $^{(4)}$ & 24.0 & 326 & 0.20 & 0 & 0.056 & $34.0^{\circ}$ & 0 \\
\hline 2 (blasted) $^{(4)}$ & 24.0 & 556 & 0.20 & 0 & 0.138 & $50.0^{\circ}$ & 0 \\
\hline 3 (blasted) $^{(4)}$ & 24.0 & 350 & 0.20 & 0 & 0.185 & $40.0^{\circ}$ & 0 \\
\hline 4 (blasted) $^{(4)}$ & 14.0 & 280 & 0.20 & 0 & 0.042 & $24.0^{\circ}$ & 0 \\
\hline 5 (blast slot) $)^{(4)}$ & 2.0 & 30 & 0.20 & 20 & 20.0 & $65.0^{\circ}$ & 0 \\
\hline
\end{tabular}

Notes: (1) $\mathrm{t}=$ large-scale rock mass tensile strength

(2) $\left(c_{p, r}, \phi_{p, r}\right)=$ equivalent Mohr-Coulomb shear strength parameters from Generalised Hoek-Brown envelope over the stress range of interest for modelling, adjusted based on experience and judgement Elastic-perfectly plastic material behaviour assumed, peak $=$ residual strength for rock mass

(3) $\mathrm{D}$ = plastic dilatancy angle set to zero because of elastic-perfectly plastic material behaviour model

(4) Effects of blasting simulated by reducing modulus of elasticity and creating a far-field slot with reduced unit weight but purely elastic response (see text for additional explanation)

Table 4 Rock mass interface parameters for base-case numerical modelling

\begin{tabular}{lccccc}
\hline Interface Location & $\mathrm{ks}^{(1)}(\mathrm{MPa} / \mathrm{m})$ & $\mathrm{kn}^{(2)}(\mathrm{MPa} / \mathrm{m})$ & $\mathrm{t}^{(3)}(\mathrm{MPa})$ & $\mathrm{c}^{(4)}(\mathrm{MPa})$ & $\phi^{(4)}$ \\
\hline DYU - DYL contact & 10000 & 25000 & 0 & 0 & 15.0 \\
within DYL & 10000 & 25000 & 0.001 & 0.010 & 18.0 \\
\hline
\end{tabular}

Notes: (1) ks = interface shear stiffness

(2) $\mathrm{kn}=$ interface normal stiffness

(3) $t=$ tensile strength across interface

(4) $(c, \phi)=$ Mohr-Coulomb shear strength parameters for interface, elastic-perfectly plastic behaviour assumed

\section{Finite element modelling of highwall excavation and coal mining}

Simulation of strip mining is not simple. In general, and applicable to Saraji Mine Strip 23, mining of previous strips results in complex multi-layer dumping on the up-dip side and complex multi-level prestrip excavation on the down-dip side. In principle the full mining sequence should be simulated, but in practice this can be tedious and unnecessarily complex depending on the purpose of the modelling. As part of this study, but not reported here, sensitivity modelling was undertaken to examine the influences of many factors on the results of the numerical modelling processes. Based on the results of such simulations it was possible to greatly simplify the modelling process while being confident of capturing representative outcomes for the specific Strip 23 excavation geometry.

Part of the simulation process involves the capabilities of the modelling package. For this investigation the commercial PHASE2 code developed by RocScience was used because it is a readily available tool that has been specifically designed to simulate excavation in rock masses. One of the features of PHASE2 is the simulation of excavation and/or filling processes in stages. To model such stages the boundaries between different materials and different stages of the processes are specified using 'Material' or 'Stage' boundaries. The ultimate boundary of an excavated area is simulated using an "Excavation" boundary. Stage and Material boundaries have to satisfy in-plane force equilibrium across the boundary, but this only means that the in-plane normal and shear stress components across the boundary are locally the same. When there are differences in material behaviour across the boundary, both the in-plane stress parallel to the boundary and the out-of-plane stress may be discontinuous. This is illustrated in Figure 2. While it does not fully simulate 
the discontinuum behaviour of a mass composed of virtually rigid blocks, it does allow some "blockiness" in the stress distributions that may develop.

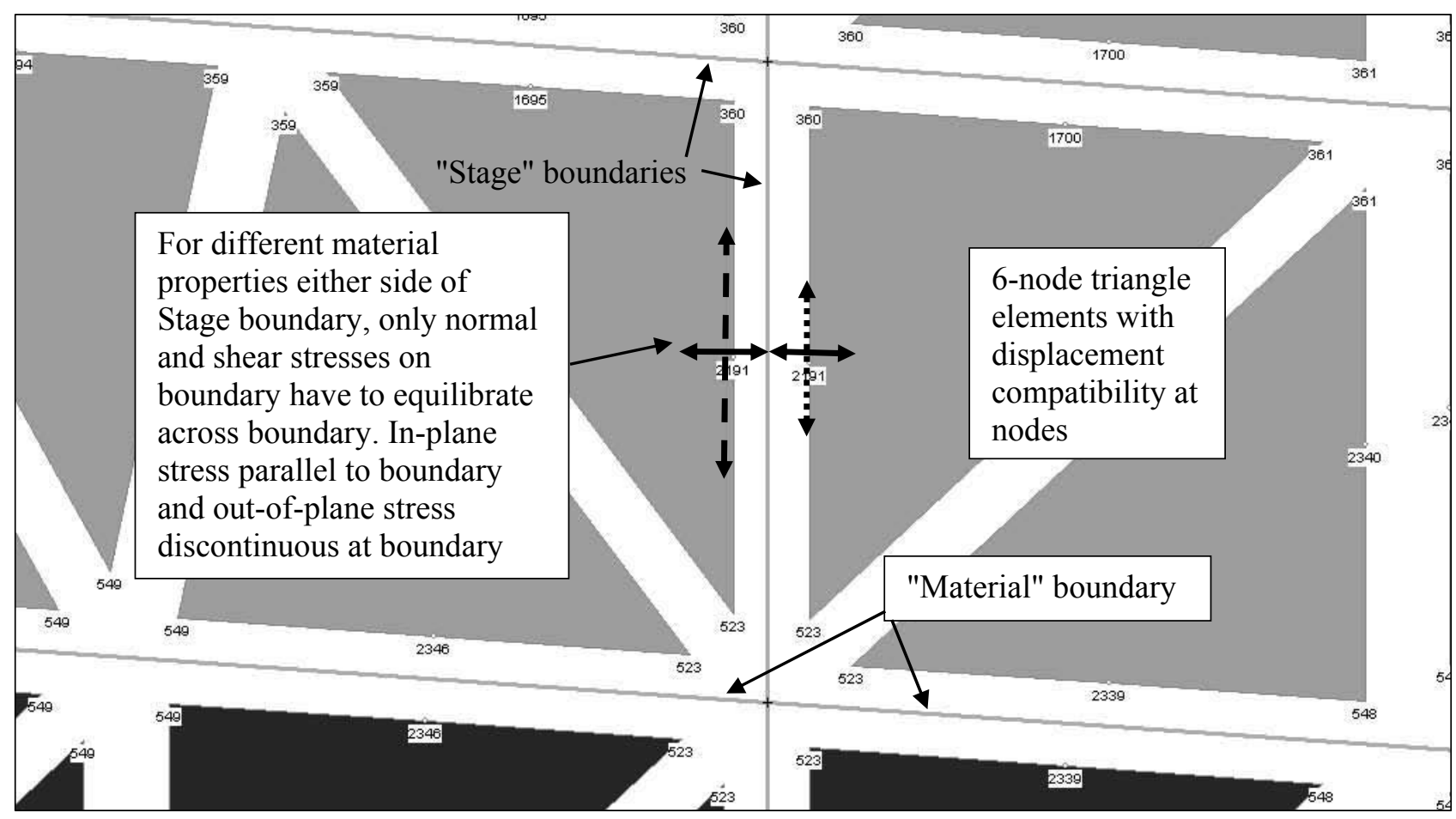

Figure 2 Finite element mesh detail showing six-node triangular elements shrunk for clarity and "blocky" stress discontinuity at "Material" and "Stage" boundary

To obtain reasonable model performance for the Saraji Mine Strip 23 situation using as simple an approach as possible, it was found to be sufficient to model the highwall excavation for the $\mathrm{K}$ - Dysart interburden interval. The lateral margins of the mesh were located at least 3 times the wall height away from the final wall position, and the base of the model was located at least the wall dimension below the slope toe.

The sequence of mining required seven analysis stages which are described in Table 5. Figure 3 shows the 2D finite element model at the stage where the coal seam overburden had been stripped but prior to coal mining. Joint elements were used at the DYU-DYL interface and at mid-thickness within the DYL layer.

Table 5 Finite element model stages for excavation sequence

\begin{tabular}{|c|c|}
\hline Stage & Description \\
\hline 1 & $\begin{array}{l}\text { Equilibrate stresses throughout mesh for "pre-mined" state based on vertical } \\
\text { lithostatic stress and horizontal/vertical stress ratio K as principal stresses }\end{array}$ \\
\hline 2 & $\begin{array}{l}\text { Simulate blasting of overburden by changing properties for blasted zone and } \\
\text { "blast slot" to create horizontal deformation component }\end{array}$ \\
\hline 3 & Excavate Unit 1 zone of "blasted" overburden \\
\hline 4 & Excavate Unit 2 zone of "blasted" overburden \\
\hline 5 & $\begin{array}{l}\text { Excavate Unit } 3 \text { zone of "blasted" overburden to expose upper boundary of } \\
\text { coal seam, and remove DYU coal layer to leave only Strip } 23 \text { part in place }\end{array}$ \\
\hline 6 & $\begin{array}{l}\text { Excavate DYU coal layer to simulate first stage of coal removal and } \\
\text { examine stress and strain response of highwall particularly near coal roof }\end{array}$ \\
\hline 7 & $\begin{array}{l}\text { Excavate DYL coal layer to simulate second stage of coal removal and final } \\
\text { stress and strain response of highwall particularly near coal roof }\end{array}$ \\
\hline
\end{tabular}




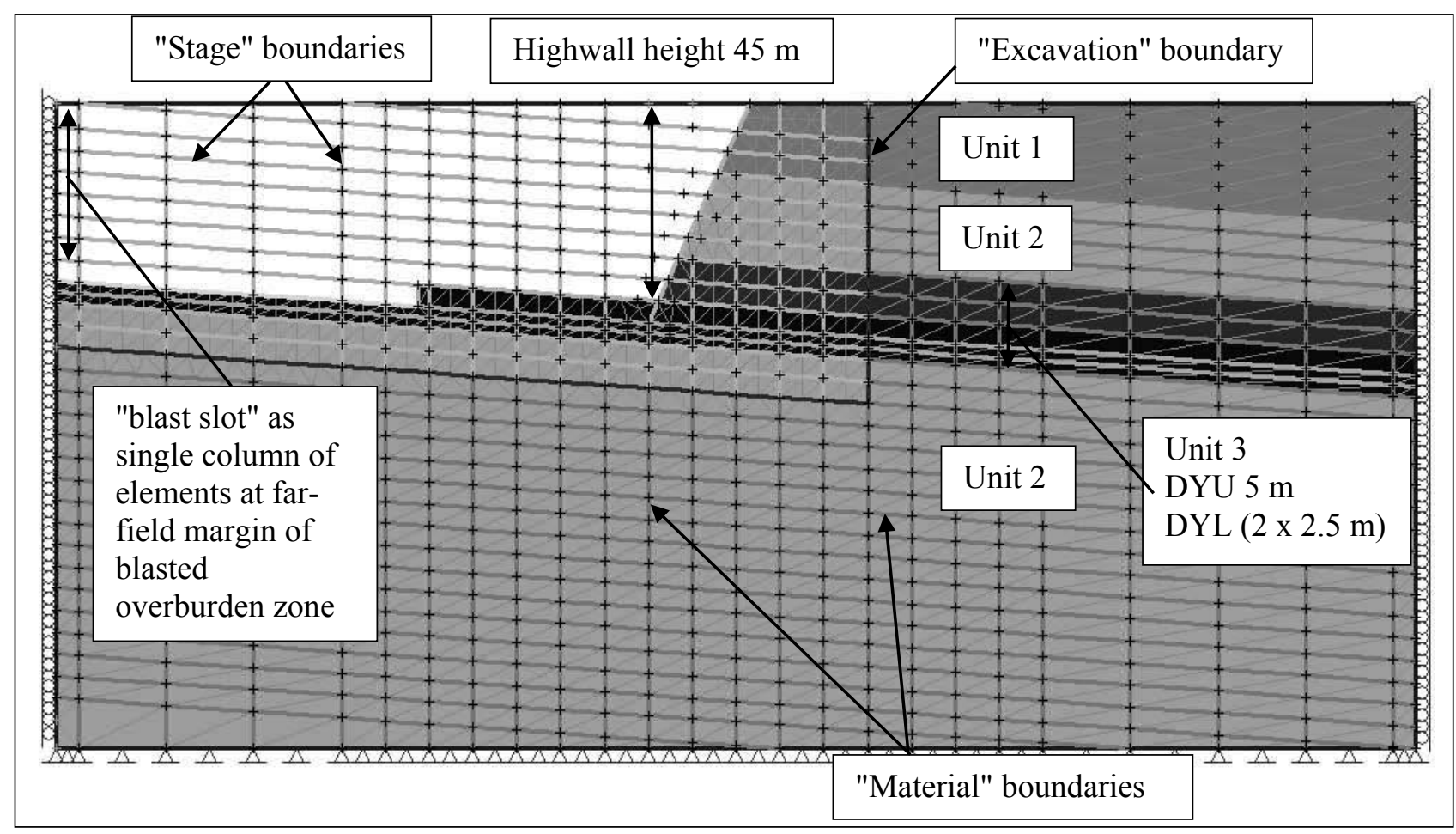

Figure 3 Finite element mesh general arrangement at Stage 5 showing features of mesh design using six-node triangular elements with both "Stage" and "Material" boundaries to simulate blockiness and "Joint" elements to simulate weak interfaces

One of the challenges to modelling is the simulation of blasting prior to excavation. The approached used for this investigation was based on previous experience, and involved simultaneously reducing the deformation modulus within the blasted materials and providing a 'blast slot' at the far-field margin of the blasted zone where material was effectively 'removed' (by reducing the density to a low value) without allowing uncontrolled deformation (by imposing very high strength within the 'slot'). This approach is not perfect but produces stress and deformation fields which were judged to be reasonable for the purpose.

The initial stress state was thought to be of major significance to the outcome of the modelling process. Virgin stress measurements made in the Ramp 12 area and indicate horizontal/vertical stress ratio K values of between 2.5 and 4.0 (BMA Coal technical staff personal communications). Because of the prior stripping, stresses close to the Strip 23 highwall were likely to have been considerably reduced by lateral extension, but the simultaneous effect of prestripping was thought to be capable of causing horizontal stresses to be 'locked-in' so there was considerable doubt about what value of K might apply in practice.

It was expected that some plastic deformation of the rock mass would develop due to the stress changes induced by the excavation sequence. Two plastic yielding processes are simulated in the PHASE2 code: shear and tension. The shear strength of the rock materials was modelled with a reasonable degree of confidence but the tensile strength presents major challenges. One estimate of effective rock mass tensile strength may be obtained through the use of the ROCLAB ${ }^{\mathrm{TM}}$ correlations. Another estimate may be made by setting the rock mass tensile strength to zero, which is equivalent to imposing a criterion that there is a pervasive network of defects that individually have no strength and which therefore limit the overall tensile stresses within the mass to zero. The latter approach was adopted for the Strip 23 investigation.

\section{Model interpretation and field behaviour}

The base-case model was based on a horizontal/vertical initial principal stress ratio $\mathrm{K}=1.5$. Other values of $\mathrm{K}$ were selected in the range from 0.5 to 3.5. The out-of-plane principal stress was also set to the in-plane $\mathrm{K}$ value. Overall deformations in response to overburden stripping and mining were computed to be in the range of 20 to $60 \mathrm{~mm}$ depending on the magnitude of the initial value of $\mathrm{K}$. 
The overall patterns of deformation and stress in response to excavation were found to be of considerable interest but will not be discussed in any detail in this paper. For all $\mathrm{K}$ values significant plastic yielding was computed. The blockiness of the model was emphasised by the discontinuities in stress contours at material boundaries. For even the most extreme values of K, 0.5 and 3.5, there were no indications of instability or collapse. PHASE2 incorporates a facility for finite element computation of slope stability using the SSR technique but this was not evaluated at the stage of investigation reported herein.

Figure 4 is a plot of the principal stress ratio at the final stage of coal mining. The extent of yielding of the two contact surfaces within the DYU - DYL seam interval were not shown on this figure for clarity, but extended for a distance of approximately the highwall height behind the toeline.

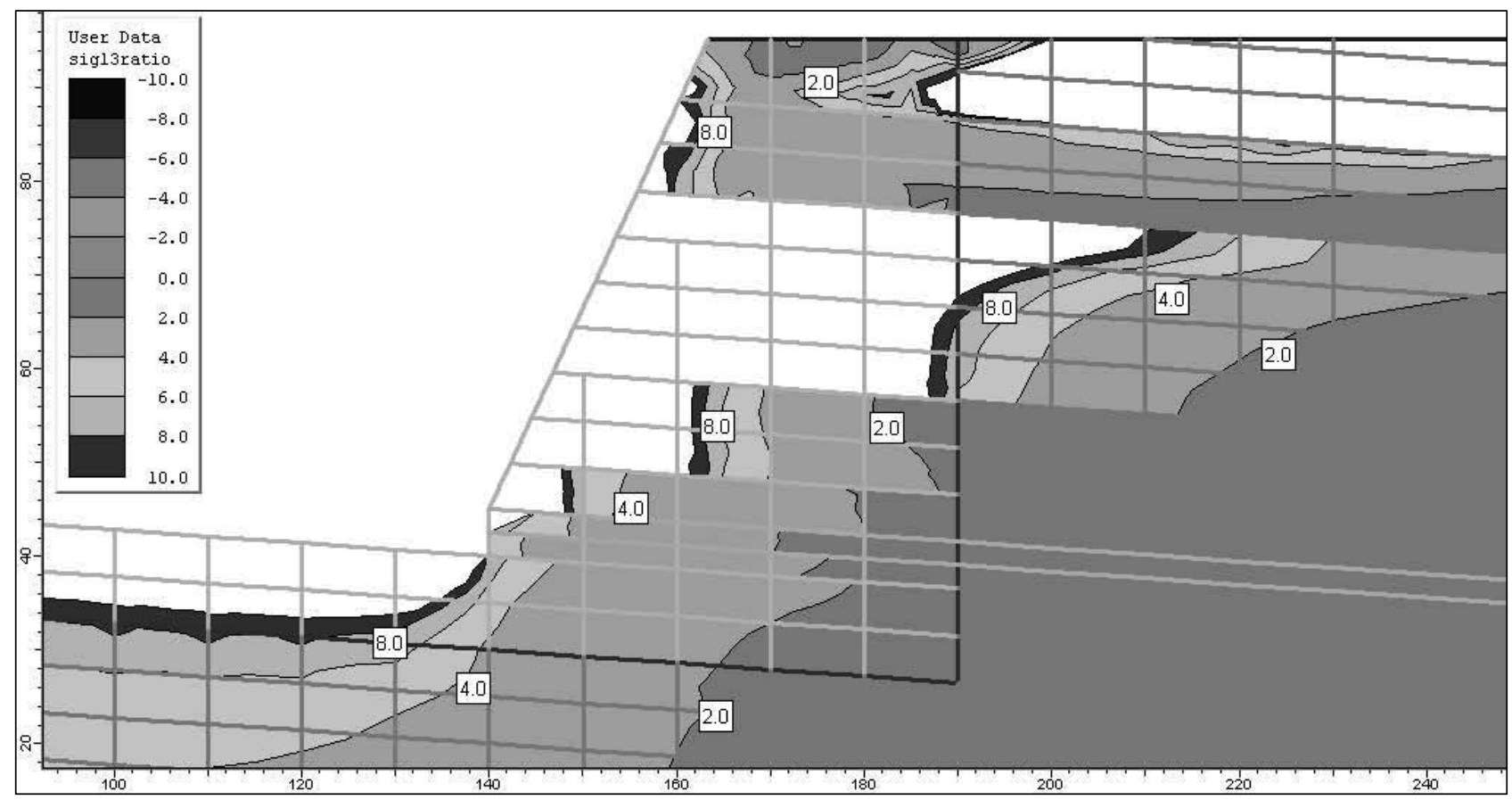

Figure 4 Example results for final mining stage principal stress ratio for $K=1.5$ and no-tension criterion applied. Note discontinuities at material boundaries and extension zone behind highwall crestline (line framework shows location of "Material" and "Stage" boundaries)

The most striking feature of the result shown on Figure 4 is the extreme range of principal stress ratio results within the area of interest. Very high principal stress ratios are indicated within the immediate floor of the excavation and behind the crestline of the slope. High principal stress ratios particularly with near-zero minimum stresses are interpreted as indications where brittle fracture is most likely to develop. The surprising aspect is that the strongest and stiffest material, the Unit 2 sandstone, was predicted to have a zone of extreme principal stress ratio for an unexpectedly large distance behind the highwall face.

Sensitivity analyses were also undertaken to investigate the effect of the blockiness modelling on the extent of the predicted extreme stress ratio zone. The results are not discussed in this paper, other than to report that the blockiness does have an influence on the distribution of principal stress ratio which is interdependent with the initial principal stress ratio K and the arrangement of 'material' and 'Stage' boundaries.

By comparing the predicted zone of extreme principal stress ratio with the physical features of the failure shown in Figure 1, it may be concluded that Figure 4 does show many of the features observed in the actual post-failure condition of the slope. However, the modelling indicated plastic yielding but not instability, and it is likely that additional factors would have to be considered if the numerical modelling was to be used for back analysis of the failure condition.

Figure 5 is a plot of the computed ratio of extensional strain to ESFC value. Values of this ratio that are 1.0 or greater indicate conditions where extension strain fracturing is predicted to occur. Also shown on this figure are rosettes indicating the directions and relative magnitudes of the principal stresses $\sigma_{1}$ and $\sigma_{3}$. Since 
this figure shows changes from the Stage 1 equilibration, these principal stress rosettes are likely to provide a very good guide to the directions in which extension fractures would propagate, that is along the direction of the longest arm of the rosette.

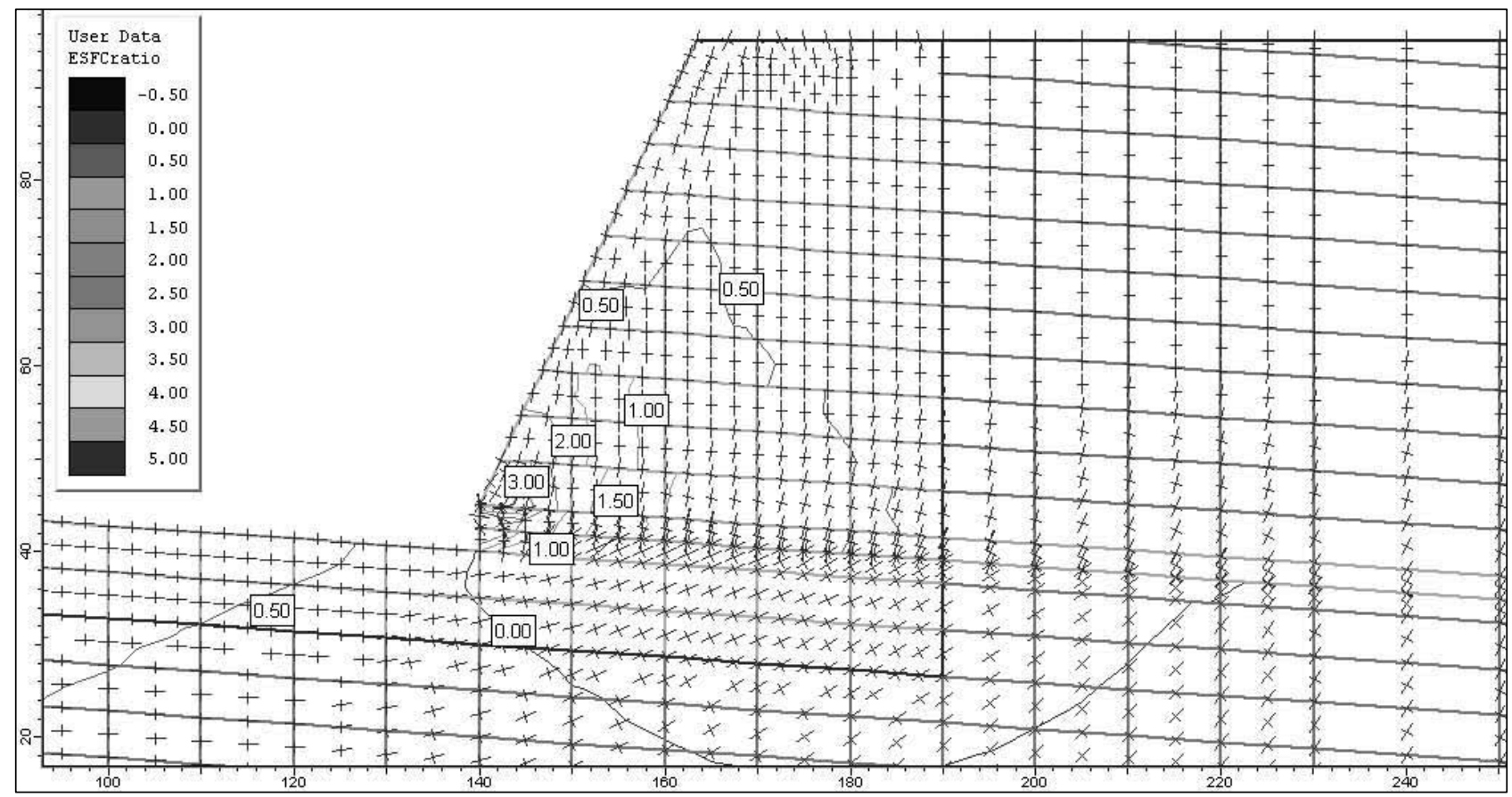

Figure 5 Example results for final mining stage ESFC Ratio $\left(\varepsilon_{3} /\right.$ ESFC) for an ESFC value of 0.00025 and initial principal stress ratio $K=1.5$. Superposed on this plot are the principal stress orientation rosettes, where the greatest magnitude shows the most likely orientation of extension strain fracturing indicated for ESFC ratios of 1.0 or greater

The intention of this paper was to model conditions under which composite failures have been experienced. The results in Figure 5 do not show failure conditions, however they do show that extension strains are likely to develop within the upper part of the coal seam and definitely within the immediate roof overburden. As a rule of thumb, the onset of extension strain fracture in laboratory conditions occurs at a strain or stress level that is about $33 \%$ of the condition under which failure and/or runaway deformations develop. As a rough guide, ESFC Ratio values in excess of 3.0 might logically be associated with deformation where runaway fracturing could occur. Figure 5 indicates that such levels were predicted to occur at and near the immediate roof of the coal seam for a principal stress ratio $\mathrm{K}=1.5$. Taken together with the results indicated in Figure 4, the outcome of the modelling is to predict that extensional strain fracturing processes are likely to occur within a zone of the highwall where composite failures have been observed.

Most significantly, and not surprisingly, the orientations of the extensional fracturing predicted by the above modelling process coincide with the orientations of the steep outward-dipping defects observed in association with many composite failures. The modelling process, while limited and simplified compared to the real rock mass, appears to be capable of demonstrating that composite failure of bedded rock masses involves processes of extensional brittle fracturing.

\section{Conclusions}

So far in this paper the existence and numerical value of an appropriate extension strain fracture criterion for bedded sedimentary rocks has not been discussed. The ESFC value of 0.000250 quoted in the Figure 5 caption is not accidental or without foundation (Stacey 1981). The results reported in this paper are not sensitive to this value, and similar patterns and magnitudes of results may be determined by undertaking sensitivity studies using the approach outlined in this paper. 
The ESFC concept is neither new nor without foundation. It does not offer a simple fix or a simple prediction for complex design assessments. It is an indicator of a process, and the results reported in this paper are believed to provide credible support for the hypothesis advanced in Section 2. Clearly much more investigation is required before such modelling can be extended to provide reliable prediction of the composite failure process. It is equally clear that the role of stress conditions, blockiness, and extensional strain fracturing must be taken into account in order to understand the triggers for composite failure.

\section{Acknowledgements}

Studies described in this paper form part of project C15033 funded by the Australian Coal Association Research Program (ACARP) and supported primarily by BHP Billiton Mitsubishi Alliance Coal (BMAC). ACARP industry monitors have provided valuable project advice, while staff of BMAC Saraji Mine have provided support for project fieldwork. Essential support and advice have also been provided by ISS International and Groundprobe for the field component of the project and from RocScience for the computational aspects of the project.

Professor Dick Stacey of the University of the Witwatersrand originated the extension strain fracture criterion as a conceptual but practical framework for understanding the inelastic response of rock to stress change. Dick's emphasis on combining rigorous understanding with a practical engineering approach has provided guidance and advice to the authors in the pursuit of the project. While acknowledging the key role of BMAC in the project, the opinions and results expressed in the paper are the sole responsibility of the authors.

\section{References}

Dight, P.M. (2006) Pit wall failures on "unknown" structures. Proceedings International Symposium on the Stability of Rock Slopes in Mining and Civil Engineering Situations, Cape Town, The South African Institute of Mining and Metallurgy, pp. 11-29.

Fujii, Y., Kiyama, T., Ishijima, Y. and Kodama, J. (1998) Examination of a rock failure criterion based on circumferential tensile strain. Journal of Pure and Applied Physics, Birkhäuser Verlag, Volume 152, pp. 551-577.

Hillis, R.R., Enever, J.R. and Reynolds, S.D. (1999) In situ stress field of eastern Australia. Australian Journal of Earth Sciences, Volume 46, Number 5, pp. 813-825.

Mandl, G. (2005) Rock Joints The Mechanical Genesis. Springer, Berlin, p. 221.

Mogi, K. (2007) Experimental Rock Mechanics. Taylor and Francis, London, p. 358.

Simmons, J.V. and Simpson, P.J. (2006) Composite failure mechanisms in coal measures rock masses - myths and reality. Proceedings International Symposium on the Stability of Rock Slopes in Mining and Civil Engineering Situations, Cape Town, The South African Institute of Mining and Metallurgy, pp. 31-49.

Stacey, T.R. (1981) A simple extension strain fracture criterion for fracture of brittle rock. International Journal of Rock Mechanics and Mining Sciences, Volume 18, pp. 469-474.

Stacey, T.R. (2006) Considerations of failure mechanisms associated with rock slope instability and consequences for stability analysis. Journal of the South African Institute for Mining and Metallurgy, Volume 106, Number 7, pp. 485-493. 